\title{
Acute Large Bowel Obstruction Following Late Sequelae of Traumatic Diaphragmatic Hernia
}

\begin{abstract}
A 33 years old patient was admitted in a Military Hospital with the features of acute large gut obstruction. Exploratory laparotomy was done and peroperatively the obstruction was seen in the transverse colon and obstruction seemed to be carcinoma of transverse colon with involvement of left hemidiaphragm which was not negotiable. Transverse loop colostomy with decompression of bowel was done. Subsequently the patient was transferred to tertiary level hospital in Dhaka. In the tertiary hospital, the patient developed left sided massive pleural effusion. With relevant investigation the condition was diagnosed as left sided diaphragmatic hernia. Thoracotomy was done and herniorrhophy was performed after reduction of the content. Postoperative management was stormy and eventful but the condition improved gradually and the patient was discharged in proper time. The patient had history of chest trauma due to RTA he met 4 years back and he received hospital indoor management for $3 \frac{1}{2}$ months. The patient was asymptomatic and leading normal active military life before 2 nd time admission for acute intestinal obstruction. The patient had past history of trauma to left chest wall 4 years back, presented with acute large gut obstruction and there was diagnostic dilemma. There were management difficulties and post operative events were stormy. The aim of this reporting is to highlight all of these.
\end{abstract}

Key-words: Acute large bowel obstruction, Late sequelae, Traumatic diaphragmatic hernia.

\section{Introduction}

Abdominal viscera herniating through defects in the diaphragm produce diaphragmatic hernia. The majority of diaphragmatic hernias in adults are either standard hiatal hernias or those presenting acutely due to traumatic disruption of the diaphragm which present months to years after the traumatic (or surgical) event'.

\section{Case Report}

Corporal Md Rasheduzzaman, 33 years of age was admitted in $\mathrm{CMH}$ Ghatail on 27-10-10 with the complaints of diffuse abdominal pain and distension with absolute constipation for 03 days. There was occasional vomiting. The symptoms were gradually aggravating. There was no history of haematemesis, melena, fever or dysuria. He had a past history of hospitalization for $3 \frac{1}{2}$ months due to road traffic accident while he sustained fracture of 3-9 ribs and clavicle on the left side 04 years back. On examination his built was average, haemodynamically stable, abdomen was moderately distended and diffusely tender, bowel sound was hyperactive and digital rectal examination was normal. Plain $X$ ray abdomen antero-posterior (AP) view showed multiple gas distended bowel loops (Figure-1). He was labeled as a case of acute intestinal obstruction and was treated conservatively, but he developed gradual abdominal distention and bowel sound became sluggish. Repeat plain X-ray abdomen AP view on 30-10-10 shows hugely distended transverse colon. On the same day exploratory laparotomy was done and found hugely distended small intestine up to transverse colon. A mass was found about $10 \mathrm{~cm}$ proximal to left colic flexure obstructing the lumen. The mass was fixed with left hemidiaphragm. Obstruction could not be negotiated. Per operative impression was large gut obstruction due to a growth in the transverse colon. Biopsy could not be taken as margin of the growth could not be visualized. Transverse loop colostomy with decompression of bowel was done. On 02-11-10 the patient was transferred to $\mathrm{CMH}$ Dhaka for further evaluation and management. On the next day patient had left sided chest pain and respiratory distress. X-ray chest postero-anterior (PA) view showed homogenous opacity in the left lung field with left sided pleural effusion (Figure-2). Bronchoscopy was done and found thick mucosal plug in the lumen of left bronchus, otherwise normal findings. On 04-11-10 about 01 liter of haemorrhagic pleural fluid was aspirated. On 06-11-10 re-aspiration of pleural fluid and on 07-11-10 tube thoracostomy was done. Cytological examination of pleural fluid confirmed no malignant cell. Colonoscopy tried but could not be passed beyond left colic flexure. CT scan of abdomen and chest suggestive of diaphragmatic hernia on the left side (Figure-3). On 10-1110 left postero-lateral thoracotomy was done. Reduction of the protruded transverse colon with excision of omentum and repair of diaphragm was done. On 11-11-10, patient developed haematemesis and bleeding through colostomy. Exploratory laparotomy was done immediately and found massive fresh and clotted blood in stomach, small intestine and colon. Active bleeding from gastro-duodenal artery was identified and managed accordingly. Subsequently chest condition improved. Colostomy was closed on 18-01-11 and patient was discharged on 05-02-11. Patient was reviewed after 01, 03, 06, 12 months and found to be asymptomatic with good respiratory and bowel function. Wound healed leaving healthy scar.

1. Lt Col Md Mahboob Hasan, MBBS, FCPS, Diploma (Hepatobiliary Surgery), Classified Specialist in Surgery \& Hepatobiliary Surgeon, CMH, Dhaka (E-mail: drmhasanhbp@gmail.com) 2. Maj Gen MA Baqui (Retd), MBBS, FCPS, Ex-Professor of Surgery, AFMC, Dhaka 3. Lt Col Farzana Rahman, MBBS, MCPS, FCPS, Classified Specialist in Radiology, CMH, Cumilla 4. Lt Col Merajul Hasan, MBBS, FCPS, Classified Specialist in Surgery, CMH, Bogura. 


\section{Discussion}

Diaphragmatic hernia may be congenital or acquired. Acquired hernias can be traumatic or iatrogenic ${ }^{2}$. Abdominal organ herniation due to diaphragmatic injury was first described by Sennertus in $1541^{3}$. They occur following motor vehicular accidents, falls and stabs or after upper abdominal surgeries ${ }^{4}$. It is found in up to $5 \%$ of patients with trauma ${ }^{5}$. In asymptomatic patients with thoraco-abdominal stab wounds, the risk of an occult diaphragmatic injury is approximately $7 \%$, and a mortality rate of $36 \%$ following delayed recognition ${ }^{6}$, Approximately $68.5 \%$ of these tears occur on the left side; $24.2 \%$ are on the right and $1.5 \%$ are bilateral. The left-sided predominance is presumed to be attributable to the relative weakness of the left hemidiaphragm and the protective effect of the liver on the right side ${ }^{7}$.

Patients with delayed diaphragmatic herniation frequently present months to years after the initial injury with manifestations of visceral herniation, incarceration, obstruction, ischemia from strangulation, or perforation ${ }^{8}$. Some patients may remain symptom-free even at the time of diagnosis. The major reasons for delay in symptoms in the patient may have occurred due to the temporary "plugging" of the diaphragmatic defect by the omentum, which was not able to prevent symptomatic visceral herniation from occurring later ${ }^{7}$. Often, these cases present with chronic chest or abdominal symptoms and rarely present as an emergency like the presented case. Diagnostic tools include plain thoracic radiographs, upper gastrointestinal contrast studies, diagnostic peritoneal lavage, fluoroscopy, ultrasound, computed tomography, magnetic resonance imaging, laparotomy, and videoassisted thoracic surgery. There is no single definite modality advocated for diagnosing traumatic diaphragmatic hernias. Interestingly, only $25-50 \%$ of initial chest films are diagnostic ${ }^{7}$.

Diaphragmatic hernia must be managed surgically. There is controversy as to whether a laparotomy or thoracotomy should be done. Thoracotomy enables the division of the adhesions between thoracic and herniated abdominal viscera while in laparotomy bowel resection and anastomosis, if needed, is easily performed. Sometimes a combined approach may be necessary. The contents usually include mostly the small intestine and portion of large intestine. The incarcerated loops of bowel should be carefully dealt with. Traumatic diaphragmatic hernia must be restored by non-absorbable sutures or synthetic grafting ${ }^{9}$. More recently, successful laparoscopic and thoracoscopic repairs of the hernia have both been described. Some authors have also described hand assisted thoracoscopic repair of hernia ${ }^{10}$.
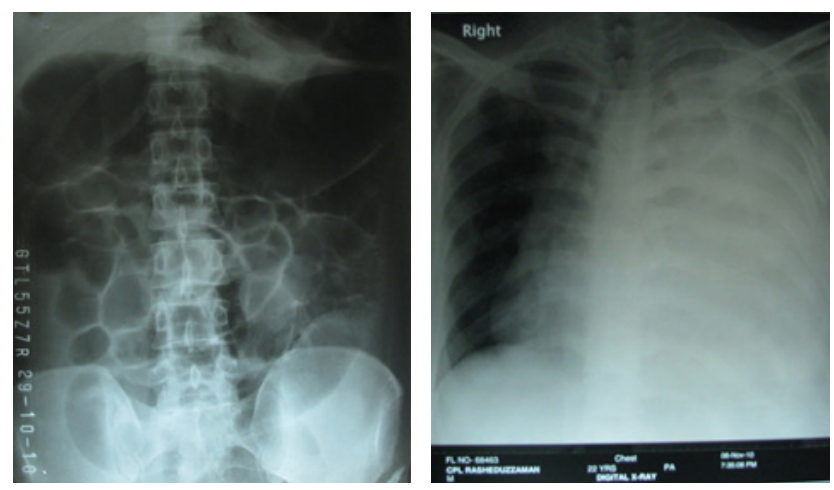

Figure-1: X-ray abdomen A/P view

Figure-2: X-ray Chest P/A view

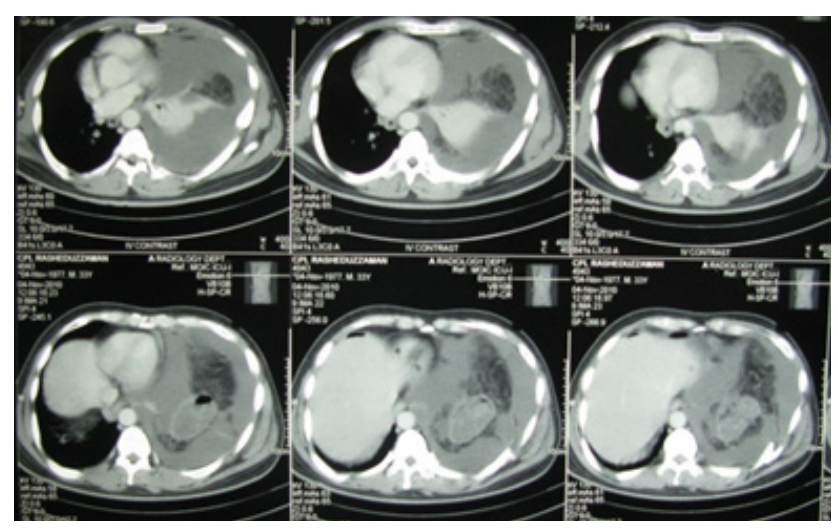

Figure-3: CT scan of abdomen.

\section{Conclusion}

Early diagnosis of diaphragmatic rupture after trauma may be difficult, and delayed diagnosis may result in increased morbidity and mortality. It is to be emphasized that, though rare, delayed presentation of a diaphragmatic hernia should be considered in patients with prior history of trauma involving either the lower chest or upper abdomen presenting with acute signs of intestinal obstruction, especially when a conventional plain X-ray reveals some abnormal findings.

\section{References}

1 A Gunduz, S Turedi, S Turkmen. An unusual presentation of diaphragmatic hernia, Emerg Med J 2008; 25:54.

2 Tsuboi K, Omura N, Kashiwagi $\mathrm{H}$ et al. Delayed traumatic diaphragmatic hernia after open splenectomy: Report of a case. Surgery Today 2008; 38(4):352-4.

3 Turhan K, Makay O, Cakan A et al. Traumatic diaphragmatic rupture: Look to see. Eur J Cardiothorac Surg 2008; 33:1082-85.

4 Onakpoya U, Ogunrombi A, Adenekan A et al. Strangulated Tension Viscerothorax with Gangrene of the Stomach in Missed Traumatic Diaphragmatic Rupture. ISRN Surg 2011; 2011:458390.

5 Hoffmann B, Nguyen H. Diaphragmatic Laceration After Penetrating Trauma Direct Visualization and Indirect Findings on Focused Assessment With Sonography for Trauma in the Emergency Department. JUM 2009; 28(9):1259-63.

6 Leppäniemi A, Haapiainen R. Occult diaphragmatic injuries caused by stab wounds. J Trauma 2003; 55(4):646-50.

7 Tang CT, Liu MY, Tang CT. Delayed presentation of a traumatic diaphragmatic hernia. SIGNA VITAE 2011; 6(1):53-5.

8 Lal S, Kailasia Y, Chouhan S et al. Delayed presentation of post traumatic diaphragmatic hernia. JSCR 2011; 7:6.

9 Ozkan OV, Semerci E, Yetim I et al. Delayed diagnosis of traumatic diaphragmatic hernia may cause colonic perforation: A case report. Cases Journal 2009; 2:6863.

10 Kumar A, Maheshwari V, Ramakrishnan TS et al. Caecal perforation with faecal peritonitis- unusual presentation of Bochdalek hernia in an adult: A case report and review of literature. World Journal of Emergency Surgery 2009; 4:16. 